\title{
Una aproximación teórica sobre la gestión del conocimiento
}

A Theoretical Approach to Knowledge Management

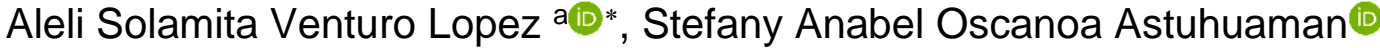 \\ aUniversidad Peruana Unión, Lima, Perú
}

\section{Resumen}

En este presente artículo se tiene como objetivo desarrollar los aspectos teóricos de la gestión de conocimiento mediante sus orígenes y dimensiones. En este método se utilizó el Mendeley como aun acercamiento para las citaciones y las referencias. Los inicios de la gestión de conocimiento surgieron en los años 70 muestra a lo largo de la historia la concientización sobre conocimiento como un medio de información, en los años 80 las organizaciones pusieron programas productivos basadas en iniciativa de mejora de calidad, en los años 90 se identifica la gestión del conocimiento con las destrezas de las personas para conocer y emplear y manejar la información utilizando la tecnología y el intercambio de conocimiento y en la actualidad se implementa la gestión del conocimiento $(\mathrm{GC})$ ya que se origina un impacto positivo en las organizaciones al enlazar conocimiento y estrategia. Las dimensiones de la GC son desarrollo de programas de gestión del conocimiento da referencia a las habilidades de la organización para fomentar programas de gestión del conocimiento, uso de transferencia de conocimiento da informe a la eficacia y eficiencia de las habilidades de GC permite diseñar mejores estrategias, conocimiento interno se da mediante la iniciativa y motivación que patenta el propio trabajador, así como también hace referencia a las técnicas de la empresa para el progreso de nuevos conocimientos, conocimiento externo son habilidades para desarrollar conocimiento dentro de la empresa internamente y específico también permite la innovación. La relevancia de este estudio radica a partir de teórico y conceptualizaciones que permita realizar otros elementos teóricos más excautivos.

Palabras Clave: Conocimiento; habilidades; competividad; estrategias

\section{Abstract}

The objective of this article is to develop the theoretical aspects of knowledge management through its origins and dimensions. In this method, Mendeley was used as an approach for citations and references. The beginnings of knowledge management emerged in the 70's

\footnotetext{
${ }^{*}$ Autor de correspondencia:

Tel.: +51960367202

E-mail: aleliventuro@upeu.edu.pe

DOI: https://doi.org/10.17162/riva.v8i1.1634

Recibido: 09/10/2021 Aceptado: 8/12/2021
} 
showing throughout history the awareness of knowledge as a means of information, in the 80's organizations put productive programs based on quality improvement initiative, in the 90's knowledge management is identified with the skills of people to know and use and manage information using technology and knowledge sharing and nowadays knowledge management $(\mathrm{KM})$ is implemented as it originates a positive impact on organizations by linking knowledge and strategy. The dimensions of $\mathrm{KM}$ are development of knowledge management programs refers to the skills of the organization to promote knowledge management programs, use of knowledge transfer reports the effectiveness and efficiency of KM skills allows to design better strategies, internal knowledge is given through the initiative and motivation that patents the worker himself, as well as also refers to the techniques of the company for the progress of new knowledge, external knowledge are skills to develop knowledge within the company internally and specific also allows innovation. The relevance of this study is based on theoretical and conceptualizations that allow other more exhaustive theoretical elements.

Keywords: Knowledge; skills; competitiveness; strategies

\section{Introducción}

Hoy en día las organizaciones presentan una posición que trata en emplear información y constantemente es más abundante y difícil de organizar (Tello et al., 2016), es por ello por lo que las capacidades distintivas en la gestión del conocimiento (GC) y el desempeño innovador requiere desarrollar nuevo conocimiento y nuevas competencias, mediante la compra de tecnología y la interrelación con otras organizaciones (Lapiedra \& Alegre, 2005). Según Ershaghi et al. (2016) también requiere comprender los recursos y la idoneidad, además se aplica en la generación donde las alternativas de solución para evitar dichos problemas que afrontan en la sociedad (Rodríguez \& Dominguez, 2005). Asimismo, se requiere "implementar nuevas habilidades administrativas, de proyecto empresarial o de conexión con equipos de interés para la mejora de la innovación" (Pérez, 2012, p. 2).

La gestión del conocimiento permite obtener ventajas competitivas ya que facilitan la creación en el proceso de la innovación (Drucker, 2012), después de todo las organizaciones crean habilidades de gestión del conocimiento (GC) para que sea de forma más veloz y eficiente, al respecto, el conocimiento es esencial de las empresas para producir beneficios, por ende, acceder a renovar y mantener de forma constante las tenencias de las ventajas competitivas en el mercado (Rodríguez \& Dominguez, 2005). Por su parte, Sánchez et al., (2007) desarrolla nuevo instrumento que añade la flexibilidad y la posibilidad de respuesta interna y externa a la innovación, es importante tener una actitud buena con ganas de aprender de forma firme, con posibilidades de aclarar, razonar, planificar y así poder emular más efectivamente y lograr el éxito. 
Los antecedentes de este estudio evidencian de dos perspectivas científicas los resultados de la gestión de conocimiento. Se encontró evidencia empírica sobre la gestión del conocimiento que lo relacionan con otras variables (Lapiedra \& Alegre, 2005). Por otro lado, se evidencian a través de una perspectiva teórica de la gestión del conocimiento (GC) (Franch \& Mayelin, 2016, Rodríguez y Joaquiin, 2009). Por la tanto, estos estudios pocos se centran en describir las teorías y conceptos sobre la gestión del conocimiento, por lo que mereció realizar un estudio que se aproxime a la revisión teórica sobre dicho constructo, además, conocer que teoría es la más utilizada sobre la gestión del conocimiento y sus dimensiones.

\section{Desarrollo o Revisión}

\subsection{Origen teórico de la gestión del conocimiento}

El origen de gestión del conocimiento (GC) se dio con Peter Drucker en el año 1970, donde pudo describir acerca de las personas que tiene cuya habilidad y experiencias de conocimientos, ya que son importantes en la organización. En los años 80 diversos autores dieron a conocer un desfase en el desarrollo de gestión de conocimiento (GC) por la falta teórica integrada y preparación profesional, en la etapa de los años 90, aparece el término gestión del conocimiento (GC) que consiste en la capacidad de crear conocimientos y por lo tanto se obtendrá nuevos productos, servicios y sistemas. Sin embargo, en el año 2000 comenzó a obtener notablemente la gestión narrativa un entendimiento en las compañías, incrementando la competitividad de las organizaciones (Rodríguez \& Dominguez, 2005).

Arceo (2009) menciona a partir de 1970 la gestión del conocimiento (GC), se ha venido manifestando y concientizando el conocimiento como recurso de la información. Rodríguez \& Dominguez (2005) plantearon que es "una organización donde puede adquirir más información que un individuo, por tener a diferentes miembros trabajando en varios experimentos" (p.4), esta información pretende que sea usada por la organización, ya que la falta de administración es la más grande necesidad de todos los países (Sánchez, 2006).

En el año 1980 las organizaciones pusieron programas productivos basadas en iniciativa de mejora de calidad (Lambe, 2011). Sin embargo, no se ha tomado en cuenta la teoría y práctica del "Knowledge Management", donde se percataron que la gestión de conocimiento es más amplio y profundo. Al respecto Ponjuán (2015) menciona que Druker habla sobre la falta de coherencia, derivada de una base teórica integrada, falta de preparación de los profesionales que lo practican y falta de apoyo.

En el año 1990 se reconoce que la gestión del conocimiento se originó en los Estados Unidos con el término de "Knowledge Management" que en español significa gestión del conocimiento, al respecto, Silva (2017) mencionó que en el año 1995 Nonaka Takeuchi se 
basaba en la capacidad de crear conocimientos, a través de ello crearon nuevos productos, servicios y sistemas. Asimismo, Correa-Díaz et al. (2019) menciona que en el año 1995 Gopal y Gagnon analizó las circunstancias de conocimiento actuales y futuras mediante la iniciativa de los elementos de capital intelectual de las empresas. Por su parte, Jain \& Moreno (2015) mencionan que el año 1996 se ponen en uso la regla de evaluación y diagnóstico que afirma la innovación y establece el capital intelectual, donde los estudios se caracterizan por motivar el trabajo (Tejedor \& Ane, 1998). En tanto, Meyer et al. (1997) señalaron que se reconoce la gestión del conocimiento (GC) como las destrezas de las personas para captar y conducir la información, empleando la tecnología y el cambio de conocimiento. De igual forma obtener objetivos empresariales o en relación con las personas, la tecnología y el conocimiento en las compañías (Davenport et al., 1998), que permite orientarse en la transferencia de flujos de información y estimación de las personas hacia las compañías (Chinchilla et al., 2012).

A inicios de los años 2000 Tiwana (2000) lleva a la practica una iniciativa de gestión del conocimiento (GC), donde produce un efecto en el negocio al unir conocimiento y estrategia y desigualdad entre conocimiento tácito. Al respecto Rahmad et al. (2014) menciona que en el año 2002 Sallis y Jones proponen a las organizaciones fundar y crear sus propios sistemas de gestión del conocimiento (GC). En cambio, Rodríguez y Joaquiin (2009) procuran que el compromiso de los individuos hacia la distribución de conocimiento y sobre todo enseñanza continua; por su parte Montoro (2016) señala que, a partir de 2011, el interés por este tema se dispara de forma acusada hasta el día de hoy. A continuación, se muestra la evolución de la gestión del conocimiento (GC) en tres hitos muy importantes en tiempo.

\section{Figura 1}

Evolución de la gestión del conocimiento (GC)

1970 Druker genera el termino
1990 aparece el termino de gestión del conocimiento

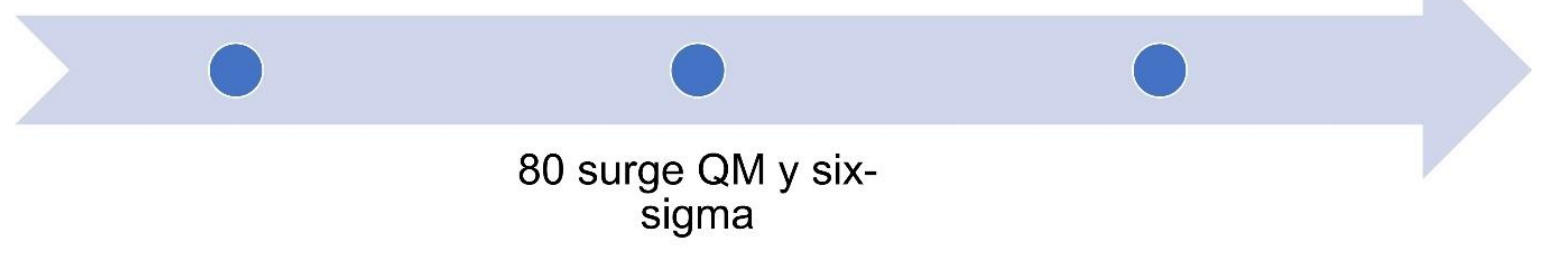

Fuente: Adaptado por Correa-Díaz et al. (2019) 


\subsubsection{Desarrollo de programas sobre gestión del conocimiento (GC)}

El desarrollo de programas sobre la gestión del conocimiento (GC) da referencia a las habilidades de la organización para fomentar programas de GC, que afecte positivamente en la creación y en el almacenamiento en la organización.

Lapiedra \& Alegre (2005) señala que el programa de GC se basa en la técnica de la organización para crear programas de gestión del conocimiento y que se obtiene mejoras en las organizaciones. Según Cepada et al (2010) facilita con las buenas prácticas que surge dentro de la organización, realiza estrategias importantes a la hora de establecer las condiciones ideales para el conocimiento. En cambio para Bueno et al. (2008) permite entender la complicación, activo de creación, incorporación , transferencia, cambió, acopio y restablecer el conocimiento organizativo, en cambio, Wiig (1994) menciona que con buenas prácticas surgen y se comparten la cultura de la organización, asimismo, de igual importancia el tener en cuenta al momento de identificar y compartir prácticas eficientes está en el aprendizaje y reutilización del conocimiento, que permitirá evitar las pérdidas del mismo (Silva, 2017).

\subsubsection{Uso y la transferencia de conocimiento (TC).}

Silva (2017) manifiesta que con el uso y la transferencia de conocimiento se refiere a la eficacia y eficiencia de las buenas practicas de gestión del conocimineto, que permite diseñar mejores estrategias para lograr que se involucren diferentes fuentes de información, ademas de tener interacción entre el conocimiento asimilado, uso de transferencia, articulación y aplicación.

Asi tambien, Quinn \& Rohrbaugh (1983) señala que la gestión del conocimiento pretende conseguir eficiencia y eficacia a partir de la planificación y el establecimiento de objetivos, con una orientación de control y un enfoque externo, beneficiando un rendimiento y la propagación de conocimientos dentro de la empresas a partir de una investigación externa, así como sus correspondientes prácticas (Lamenta \& Torres, 2015).

\subsubsection{Conocimiento externo}

La adquisición del conocimiento externo se da mediante la iniciativa y motivación que patenta el propio trabajador, haciendo referencia a sus habilidades y técnicas que usa la empresa en el proceso de gestar nuevos conocimientos, adquiriendo tecnología y la interacción con otras organizaciones, siendo una base fundamental de conocimiento en las organizaciones, en cambio, Lechuga (2008) manifiesta, para obtener los conocimientos externos debe ser mediante de la iniciativa y motivación que patenta el propio empleador, de manera que se adquiere el aprendizaje individual y grupal, por ende, desemboca nuevos 
conocimientos y beneficia a las organizaciones. Al respecto, Lapiedra \& Alegre (2005) mencionan que es la habilidad que utiliza la empresa para generar nuevo conocimiento, que le permite adquirir tecnología, ademas de la iteracción con otras organizaciones, es por ello, que Silva (2017) menciona que se requiere de un almacenamiento y transferencia para hacer uso del conocimiento, siendo esta la base fundamenta de conocimiento de las organizaciones (Lapiedra \& Alegre, 2005).

\subsubsection{Conocimiento interno}

Son habilidades para desarrollar conocimiento dentro de la empresa, que permite la innovación con el uso de tecnología y que contribuye al fortalecimiento del conocimiento organizacional, ya que vela por el progreso, objetivos y estrategias planificadas internamente, de esta manera las organizaciones tiene resultados de progreso.

Estas habilidades permiten desarrollar conocimiento interno específico en la organización (Pérez et al., 2017; Bierly et al., 2000). Son la base de conocimiento y juega un papel importante para la innovación mediante las actividades (Franch \& Mayelin, 2016), también en la parte de la innovación tecnología e incluir incorporaciones a la empresa al cual fortalece el conocimiento organizacional y vela por el progreso, objetivos y estrategias planificadas internamente, de esta manera las organizaciones tiene resultados de progreso, producto de los éxitos y los fracasos, ademas de obtener conocimientos de los especialistas de la organización. También se puede determinar, en base a su flexibilidad, su mecanismo y dirigiéndose como un mecanismo generador de nuevo conocimiento.

Para afrontar los cambios de los clientes que viene desarrollando los competidores, es necesario hacer uso de la tecnología, ya que es un factor importante para realizar nuevos productos y servicios, a través de un proceso de modelo de innovación. Asimismo, es la gestión de conocimiento que genera una ventaja competitiva prente a la competencia (Calero, 1999; Díaz et al., 2005), pues ayuda a potenciar las competencias y obtiene una ventaja sostenible (Zabala, 2012), ademas, el uso de tecnología hace que sistemáticamente pueda contribuir eficientemente en las organizaciones, para generar desarrollos de conocimiento y que contribuya en la mejora de las capacidades (Acosta, Zárate, \& Fischer, (2014).

Por otra parte González (2020) señala que la gestión del conocimiento y su dominio en la productividad, genera el mejoramiento de la articulación existente de los sistemas organizativos. Por lo que este impulsa a entender el conocimiento de la organización e incrementa la rentabilidad y crear valor agregado para el negocio (Lahaba \& Santos, 2001; García, 2006). Asimismo, desarrolla competitividad y crea conocimientos para mejorar y atribuir los procesos que ayuda en el mejoramiento de los métodos de trabajo y así se pueda 
incrementar la productividad (Martha \& Borjas, 2009). A continuación se muestra un modelo teorico de como se desarrolla la gestión del conocimineto a partir de tres grandes factores.

\section{Figura 2}

Desarrollo teórico de Gestión del Conocimiento

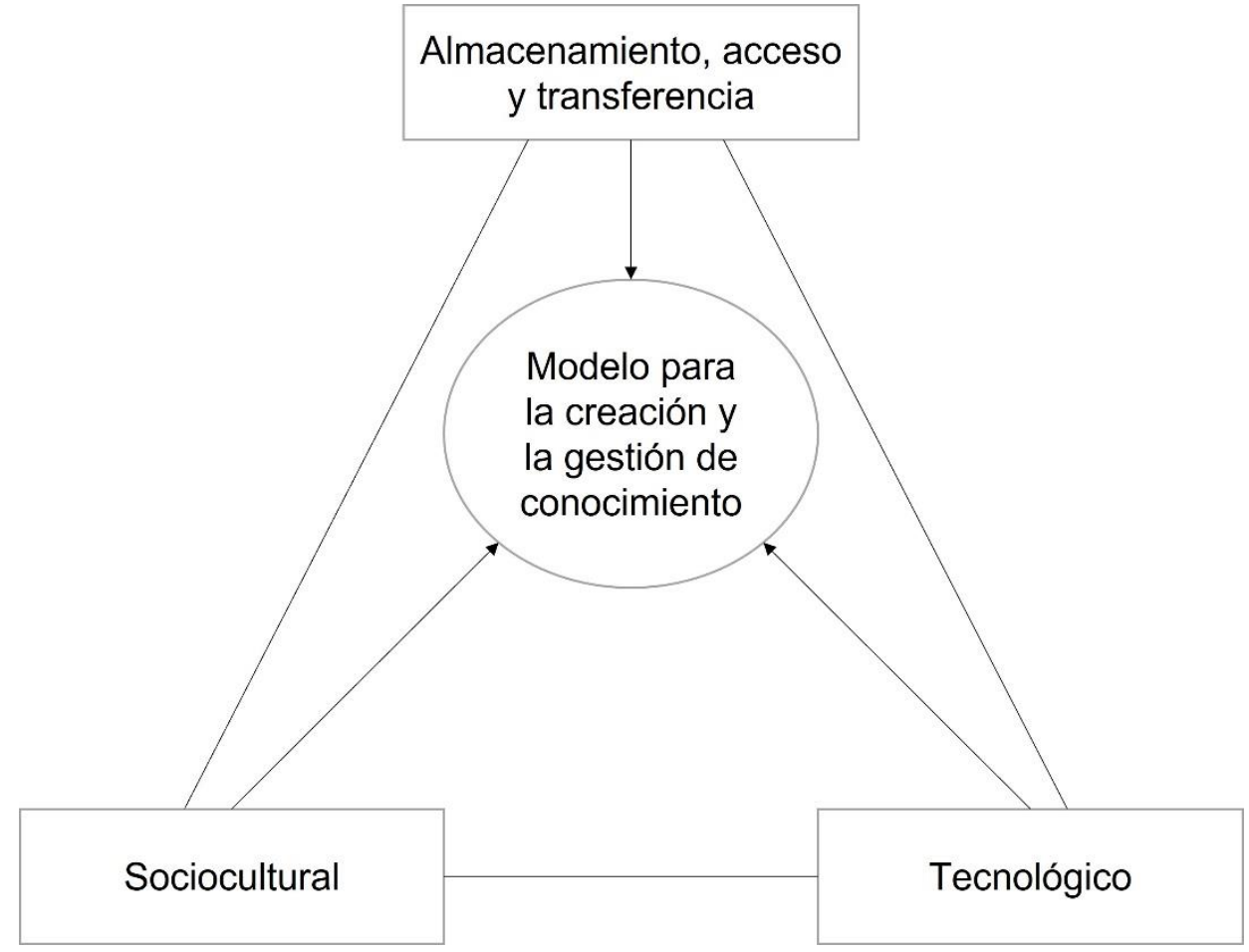

Fuente: Adaptado por Rodríguez (2006)

\section{Conclusiones}

Se concluye después de haber realizado un acercamiento teórico de la gestión de conocimiento basado en su origen y la conceptualización de sus dimensiones. El origen de gestión del conocimiento se ha desarrollado mediante Peter Drucker, al cual genera el termino, donde pudo describir acerca de las personas que tiene cuya habilidad y experiencias de conocimientos ya que son importantes en la organización.

En los años 80 dentro de diversos ejemplos teóricos de gestión de conocimiento (GC) fue desarrollado para estudiar el establecimiento, difusión y adaptación del conocimiento empresarial; para facilitar las actividades grupales e individuales que permitieron obtener cambios rápidos y el incremento de la competitividad en las organizaciones.

Producto de la revisión, se puede señalar la conceptialización de las dimensiones de la gestión de conocimiento (GC). En primer lugar, el desarrollo de programas de gestión del conocimiento $(\mathrm{GC})$ da referencia a las habilidades de la organización para desarrollar programas (GC) que afecte positivamente en la creación y en el almacenamiento en la 
organización. En segundo lugar, el uso y la transferencia de conocimiento se hace referencia a la eficacia y eficiencia de las habilidades de gestión del conocimiento (GC), permite diseñar mejores estrategias para lograr que se involucren diferentes fuentes de información, que permita la interacción entre el conocimiento asimilado, uso de transferencia, articulación y aplicación la dimensión de conocimiento externo e interno.

\section{Referencias}

Arceo, A. M. (2009). Gestion del conocimiento del concimeinto en educacion y del conocimiento en educacion y transfarmacion de la escuela. Sinéctica, 32, 1-21. http://search.ebscohost.com/login.aspx?direct=true\&db=a9h\&AN=47836179\&lang=es\& site $=$ ehost-live

Acosta, J. C., Zárate, R. A., \& Fischer, A. L. (2014). Ba: espacios de conocimiento, contexto para el desarrollo de capacidad de innovación. Un análisis desde la gestión del conocimiento. Revista Escuela De Administración De Negocios, (76), 44-63. https://doi.org/10.21158/01208160.n76.2014.797

Bierly, P. E., Kessler, E. H., \& Christensen, E. W. (2000). Organizational learning, knowledge and wisdom. Journal of Organizational Change Management, 13(6), 595-618. https://doi.org/10.1108/09534810010378605

Bueno, E., Paz, S., \& Salvador, M. (2008). Génesis, concepto y desarrollo del capital intelectual en la economía del conocimiento: Una reflexión sobre el Modelo Intellectus y sus aplicaciones. Estudios de Economía Aplicada, 26(2), 43-63. www.revista-eea.net, ref. e-26203

Calero, J. M. (1999). Sobre gestión del conocimiento, un intangible clave en la globalización. In Economía industrial. http://europa.sim.ucm.es/compludoc/AA?articulold=108633

Cepada, C., Navarro, C., Sanchez, S., \& Caro, M. (2010). El desarrollo de programas de gestión del conocimiento: las unidades de hospitalizacion a domicilio. Economía industrial, 378(1), 127-134. https://dialnet.unirioja.es/servlet/articulo?codigo=3405143

Chinchilla, N., Andrés, E. D. E., \& Díez, J. M. (2012). El Management del Siglo XXI. Reflexiones sobre el pensamiento de Javier Fernández Aguado (C. Smith (ed.); BrandSmith).

Correa-Díaz, A. M., Benjumea-Arias, M., \& Valencia-Arias, A. (2019). La gestión del conocimiento: Una alternativa para la solución de problemas educacionales. Revista Electrónica Educare, 23(2), 1-27. https://doi.org/10.15359/ree.23-2.1

Davenport, T., De Long, D., \& Beers, M. (1998). Successful Knowledge Management Projects. Sloan Management Review, 39(2), 43-57. https://www.proquest.com/openview/76ca6820cf905f6422a30b881ac0c9ea 
Díaz, D. G., de Armas, M. P., \& Valladares, I. C. (2005). Gestión del conocimiento y su importancia en las organizaciones. Ingeniería Industrial, 26(2), 37-46. https://dialnet.unirioja.es/servlet/articulo?codigo=4786692

Drucker, P. (2012). Post-capitalist society. Routledge: London and New York

Ershaghi, I., Paul, D., Hauser, M., Crompton, J., \& Sankur, V. (2016, September 6). CiSoft and Smart Oilfield Technologies. All Days. https://doi.org/10.2118/181068-MS

Franch, K., \& Mayelin, R. (2016). Las normas ISO 9000:: una mirada desde la gestión del conocimiento, la información, innovación y el aprendizaje organizacional. Cofin Habana, 10(2), 29-54. http://scielo.sld.cu/pdf/cofin/v10n2/cofin02216.pdf

García, N. N. (2006). Productividad: una propuesta desde la gestión del conocimiento. Revista Escuela de Administración de Negocios, 58, 87-105. http://www.redalyc.org/articulo.oa?id=20605807\%0Ahttp://www.redalyc.org/pdf/206/206 05807.pdf

González, S. L. (2020). Diagnóstico sobre la gestión del conocimiento para identificar el efecto en la productividad para las empresas del calzado del Barrio Restrepo. Universidad $\begin{array}{llllll}\text { Nacional Abierta } & y & a & \text { Distancia }\end{array}$ https://repository.unad.edu.co/handle/10596/39301

Jain, A. K., \& Moreno, A. (2015). Organizational learning, knowledge management practices and firm's performance: An empirical study of a heavy engineering firm in India. Learning Organization, 22(1), 14-39. https://doi.org/10.1108/TLO-05-2013-0024

Lahaba, Y. N., \& Santos, M. L. (2001). La gestión del conocimiento: Una nueva perspectiva en la gerencia de las organizaciones. Acimed, 9(2), 121-126.

Lambe, P. (2011). The unacknowledged parentage of knowledge management. Journal of Knowledge Management, 15(2), 175-197. https://doi.org/10.1108/13673271111119646

Lamenta, P., \& Torres, K. (2015). La Gestión Del Conocimiento Y Los Sistemas De Información En Las Organizaciones. Negotium, 11(32), 3-20. www.revistanegotium.org.ve

Lapiedra, R., \& Alegre, J. (2005). Gestión del conocimiento y desempeño innovador: un estudio del papel mediador del repertorio de competencias distintivas. Cuadernos de

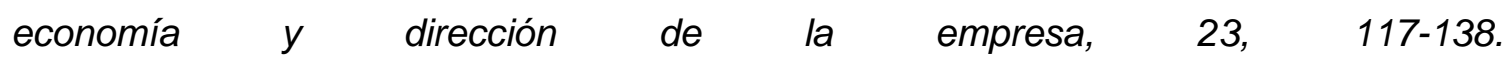
https://dialnet.unirioja.es/servlet/articulo?codigo=2162933

Lechuga, L. (2008). "El escenario de la Gestión del Conocimiento (GC) en las organizaciones productivas: estudio de caso de tres empresas en México." 1-117. http://hdl.handle.net/11191/7124

Martha, M. C., \& Borjas, M. (2009). Dra.C. Anays Más Basnuevo Ing. Yannia Adis Acosta Herrero Ms. C. Mario A. Batista Zaldívar Ms.C. Martha Morejón Borjas. 40(3), 3-13.

Meyer, D. M., Cardelli, J. A., \& Sofia, U. J. (1997). The Abundance of Interstellar Nitrogen. The 
Astrophysical Journal, 490(1), L103-L106. https://doi.org/10.1086/311023

Montoro, M. (2016). Gestión del conocimiento: orígenes y evolución. El Profesional de La Información, 25(4), 526. https://doi.org/10.3145/epi.2016.jul.02

Pérez, J. (2012). Asociaciones entre madurez de gestión del conocimiento y desempeño innovador organización y personas , e interpretación. 9(1), 1-10. http://repository.lasallista.edu.co:8080/ojs/index.php/rldi/article/view/295/141

Pérez, E. O., Toro, I. D., \& Hernandez, B. Y. (2017). Measurement of potential absorption capacity in Colombia's innovative companies. Espacios, 38(26), 1307-1321. https://doi.org/10.2307/2393553

Ponjuán, G. (2015). Knowledge management from the perspective of information sciences: Responsibilities and opportunities. Revista Cubana de Informacion En Ciencias de La Salud, 26(3), 206-216. https://www.scopus.com/inward/record.uri?eid=2-s2.084939619444\&partnerID=40\&md5=f02b9ef6acfdf193f5575431611eae0d

Quinn, R., \& Rohrbaugh, J. (1983). Spatial Model of Effectiveness Criteria: Towards a Competing Values Approach To Organizational Analysis. Management Science, 29(3), 363-377. https://doi.org/10.1287/mnsc.29.3.363

Rahmad, R. S., Rahmad Sukor, M. I., Syah, D., \& Muslihah, E. (2014). Understanding the implementation of knowledge management in high-performance schools in Malaysia. Sage OpenOpen, 4(4), 1-7. https://doi.org/10.1177/2158244014561206

Rodríguez, D., \& Joaquiin, G. S. (2009). La creación y gestión del conocimiento en las organizaciones educativas: barreras y facilitadores. Un estudio multicaso. Universidad Autónoma de Barcelona, 632. https://www.tdx.cat/handle/10803/327017

Rodríguez, Y. P., \& Dominguez, A. C. (2005). La gestión del conocimiento un nuevo enfoque en la gestión empresarial. $13(6)$, http://bvs.sld.cu/revistas/aci/\%5Cnhttp://scielo.sld.cu/pdf/aci/v13n6/aci040605.pdf

Rodríguez, D. (2006). Modelos para la creación y gestión del conocimiento: una aproximación teórica. Educar, 37, 25-39. https://www.redalyc.org/pdf/3421/342130826003.pdf

Sánchez, A. J. (2006). Innovador Maestro De La Administracion De Empresas. Cuadernos Latinoamericanos de Administración, II(2), 69-89. http://www.redalyc.org/articulo.oa?id=409634344005

Sánchez, Jiménez, Pérez, \& Carnicer. (2007). Flexibilidad e innovación: el efecto moderador de la cooperación. Revista Europea de Direccion y Economia de La Empresa, 16(4), 6688.

Silva, K. A. (2017). Aplicación del modelo de gestión del conocimiento de Nonaka y Takeuchi en el Centro Bibliográfico Nacional de la Biblioteca Nacional del Perú. https://cybertesis.unmsm.edu.pe/handle/20.500.12672/7371

Tejedor, \& Ane, A. (1998). Proyectos Logo Investigacion relativa a la capacidad de aprender 
de las empresas españolas. Boletin de Estudios Economicos, 53(164). 231-249. https://www.proquest.com/openview/3ab60647cc71baa187f201c2c87336c8

Tello, A., \& Velasco, J. M. A. P. (2016). Inteligencia de negocios: estrategia para el desarrollo de competitividad en empresas de base tecnológica. Contaduría y Administración, 61(1), 127-158. https://doi.org/10.1016/j.cya.2015.09.006

Tiwana, A. (2000). Knowledge Management Toolkit, The Amrit Tiwana Knowledge Management Toolkit, The. In Knowledge Management Toolkit. https://doi.org/10.1227/01.neu.0000403833.47211.af

Wiig, K. M. (1994). Knowledge Management Foundations: Thinking about Thinking-how People and Organizations Represent, Create, and Use Knowledge. Schema Press, Limited.

Zabala, M. (2012). La gestión de conocimientos en las organizaciones proveedoras de servicios de telecomunicaciones. Télématique, 11(1), 43-68 https://www.redalyc.org/pdf/784/78423414004.pdf 